\title{
Die Ohnmacht des Arbeitsrechts: Der Fall BMW
}

\section{Teil I: Die Beeinflussung der Betriebsratswahl und das Anfechtungsverfabren}

Zum Glück für sich und seine Arbeitnehmer in der Chefetage hat BMW Geld, sehr viel Geld sogar. Sonst hätte es dazu kommen können, daß „für den Fall der Nichtbeitreibung « eines Ordnungsgeldes in Höhe von $100000 \mathrm{DM}$ »Ordnungshaft von sechs Wochen, zu vollstrecken an einem der Vorstandsmitglieder « (ArbG Berlin, Beschluß vom 23.4. 1985, Az: 49 BVGa 2/85) Realität geworden wäre. Man stelle sich vor: Eberhard von Kuenheim, Vorstandsvorsitzender der Bayerischen Motorenwerke AG, für sechs Wochen in Ordnungshaft! Nach von Gahlen, von Brauchitsch und von Lambsdorff nun auch von Kuenheim.

Angedroht war ihm Zwangshaft schon länger, seit das Arbeitsgericht Berlin mit Beschluß vom 8. 10. 1984 (Az: I 8 Ca 142/84) gegen BMW »ein Zwangsgeld in Höhe von 20000 ,- DM, ersatzweise für je 200.- DM ein Tag Zwangshaft des Vorstandsvorsitzenden Eberhard v. Kuenheim « festgesetzt hatte. Diese gerichtlichen Beschlußformulierungen sorgten zwar für Schlagzeilen. Bislang aber blieben sie, was sie sind: bedrucktes Papier. Weder wurde v. Kuenheim in Zwangshaft genommen, noch hat der Konzern die angedrohten Ordnungsgelder bezahlt. Aber auch die zugrundeliegende Rechtspflicht, deren Durchsetzung so drastisch angedroht worden war, hat er nicht erfüllt: Und dies zu Recht, obwohl sämtliche mit der Auseinandersetzung bislang befaßten Gerichtsurteile festgestellt haben, daß BMW "in der Sache" eindeutig im Unrecht ist.

Das Recht, das so schwer durchzusetzen ist, ist der Beschäftigungsanspruch bei einem privaten Arbeitgeber, der nicht gewillt ist, das Arbeitsverhältnis fortzusetzen. Kündigt er, so bedeutet dies den Verlust des Arbeitsplatzes, der in aller Regel endgültig ist, auch wenn der Arbeitnehmer mir seiner Kündigungsschutzklage Erfolg hat: Nach empirischen Untersuchungen wurde im Jahre 1978 lediglich in 13 von 10000 Fällen ein Arbeitnehmer auf Grund eines Urteils bei demselben Arbeitgeber weiterbeschäftigt. Nun stand, nach jahrelangen heftigen Auseinandersetzungen in der arbeitsrechtlichen Rechtsprechung und Literatur über die Voraussetzungen eines Weiterbeschäfrigungsanspruchs während des Kündigungsschutzprozesses zu erwarten, daß der hierzu angerufene Große Senat des Bundesarbeitsgerichts die restriktive Linie der bisherigen höchstrichterlichen Rechtsprechung zumindest partiell aufgeben würde. Die Perspektive, noch vor dem rechtskräftigen Abschluß des Kündigungsschutzverfahrens einen vorläufig vollstreckbaren Titel auf Beschäftigung durch den Arbeitgeber - über den bisher anerkannten Fall der offensichtlich unwirksamen Kündigung hinaus - zu erhalten und durchsetzen zu können, schien nicht mehr so aussichtslos wie vordem.

$\mathrm{Daß}$ es trotz der eingangs zitierten Zwangsgeldbeschlüsse des ArbG Berlin und der zwischenzeitlich ergangenen Entscheidung des Großen Senats des BAG vom 27.2. 1985 (BB 1985, 463), die einen Weiterbeschäftigungsanspruch nach positivem erstinstanzlichen Urteil im Kündigungsschutzprozeß grundsätzlich anerkannte, 
soweit nicht kam, wußte BMW zu verhindern. Doch nicht die auch ansonsten zu beobachtende Tatsache, daß die Wirksamkeit des Rechts und seiner Veränderungen, die sie zu steigern beabsichtigen, am "Erfindungsreichtum « der Praxis ihre Grenzen findet, macht den Fall bedeutsam: Vielmehr die Art und Weise, mit der das Unternehmen vorführt, daß die Macht des Rechts dort endet, wo seine betriebliche Personalhohe1t beginnt.

\section{Der Konflikt: Die Betriebsratswabl 1984}

\section{Wablntederlage, Wablanfechtung und Kündigung}

Direkt betroffen von dieser Demonstration der Arbeitgeberprärogative gegenüber gerichtlich anerkannten arbeitsvertraglichen Verpflichtungen sind die drei Kandidaten für die Betriebsratswahl am 2. und 3. April I 984 im Berliner Motorradwerk von BMW, Rainer Knirsch, Hans Köbrich und Peter Vollmer. ${ }^{1}$ Alle drei waren Vertrauensmänner der IG Metall, Knirsch sogar Vertrauenskörperleiter und stellvertretender Vorsitzender des bis zur Neuwahl 1984 amtierenden Betriebsrats, Vollmer war freigestelltes Mitglied dieses Gremiums.

Die Wahl zu dem I s-köpfigen Betriebsrat - das BMW-Motorradwerk Berlin hatte Ende 1983 ca. 1800 Beschäftigte - wurde als Persönlichkeitswahl durchgeführt, wobei eine als "Mannschaft der Vernunft « auftretende Gruppe von Kandidaten sämtliche Plätze erreichte. Vollmer wurde mit einem Rückstand von I 2 Stimmen auf Platz I6, Knirsch auf Platz I7 gewählt, nehmen also die beiden ersten Nachrückerplätze ein, Köbrich erreichte von den insgesamt 6r Bewerbern Platz 26. Zwar waren Knirsch und Vollmer wie bei der vorausgegangenen Betriebsratswahl von ca. $35-40 \%$ der Kollegen gewählt worden, aber auf Grund der Persönlichkeitswahl sowie der Tatsache, daß sich Angestellte (ca. 20\% der Belegschaft) und Arbeiter (davon $43 \%$ Akkordarbeiter, überwiegend türkische Kollegen, und $37 \%$ qualifizierte Facharbeiter) in getrennten Abstimmungen mehrheitlich für gemeinsame Wahl entschieden hatten, langte dies nicht zum Einzug in den Betriebsrat.

Innerhalb der zweiwöchigen Anfechtungsfrist des $\$ I9 Abs. 2 S. 2 BetrVG stellten die drei unterlegenen Betriebsratskandidaten Knirsch, Vollmer und Köbrich beim Arbeitsgericht Berlin am 17.4. 1984 den Antrag, die Betriebsratswahl wegen Beeinflussung durch den Arbeitgeber für unwirksam erklären zu lassen. Der Belegschaft gegenüber begründeten sie diesen Schritt in einem am Tage zuvor vor dem Betrieb, außerhalb der Arbeitszeit, verteilten Flugblatt wie folgt:

„Berliner BMW-Werk: Wunsch-Betriebsrat des Herrn Glas

Haben die Kollegen diesen Betriebsrat wirklich gewollt?

- vergeblich suchen 400 türkische Kollegen einen Berriebsrat aus ihren eigenen Reihen.

- vergeblich suchen 700 Akkordarbetter einen Betriebsrat mit Akkord-Erfahrung.

- vergeblich suchen alle Kollegen nach einem Betriebsrat, der die praktısche Arbert der letzten 3 Jahre wetterführen kann.

Alle hatten wir eine demokratısche Betriebsratswahl gewollt. Das Ergebnis aber ist genau das Gegenteil: $40 \%$ der Beschäfrigten sind durch diesen Betrrebsrat nıcht mehr repräsentiert. Ein böses Erwachen! Niemand hat das so gewollt - außer einem: Der Werksleiter, Herr Glas, hat alle Register gezogen, um dies zu erreichen.

I Zum Konfliktverlauf, semer Vorgeschichte und Hintergründen vgl. die Broschüre: Komıtee für Grundrechte und Demokratıe (Hrsg.), Gekaufte Vernunft. Ein Lehrstück über Demokratıe und Mennungsfreihett bei BMW, Sensbachtal, Mal 1985; über den Fall $15 t$ ummer wieder auch in der überregionalen Presse berichtet worden, vgl. z.B. die Berichte in der FR vom 10.8.1984 und 4.5.1985; Der Spiegel vom I3.8.1984 und 6.5.1985; Handelsblatt vom 8/9.3. 1985. 
Massıv hat er Einfluß genommen auf die Wahl durch die Androhung, Erfolgsbeteiligung und Bonus zu streichen, wenn die Betriebsräte Knirsch und Vollmer wiedergewählt würden. Auf 2 Betriebsversammlungen hat Herr Glas persönlich und mit Flugblättern, auch in türkisch und serbokroatisch, direkt un die Wahl engegriffen.

Das geht zu weit!

Darum Wahlanfechtung!

Viele Kollegen sagen heute: aus Angst haben wir die sogenannte sMannschaft der Vernunftc gewählt, denn wir brauchen Erfolgsbeteiligung und Bonus zum Leben. Aber diesen einseitigen Betriebsrat haben wir so auch nicht gewollt.

Gesetzlich sind die Androhungen von Herrn Glas null und nichtig!

Es ist nicht möglich, Sozıalleistungen enfach zu kürzen, nur weil einem das Wahlergebnis nicht paßst. Allerdings sind die sogenannten ,freiwilligen Lesstungen immer eın Spielball der Unternehmen. Was nicht tariflich abgesichert 1st, ist eben unsicher. Die Wahlanfechtungsklage muß gegen den Betriebsrat gerichtet werden. So sieht es das Gesetz vor. Hat die Anfechtung Erfolg, dann wird von Anfang an neu gewählt. Und das Ergebnis wird mit Sicherheit anders ausfallen.

Kolleginnen und Kollegen! Es geht uns nicht darum, eine Wahl nicht verlieren zu können oder etwa mit den neuen Betriebsräten nıcht zusammenarbeiten zu wollen. Nein, es geht auschließlich gegen die absolut unfarre Einmischung von Herrn Glas, der als s Herr im Hause unsere Betriebsratswahlen bestummt hat.

Für Recht, Gerechtigkeit und Demokratıe.

Wer für eıne gerechte Sache eıntrıtt, der wird auch breite Unterstützung finden. Für die vielen guten Ratschläge und solidarıschen Wünsche sagen wir herzlichen Dank! Das hat uns in den letzten Tagen gestärkt, auch für diese Wahlanfechtung.

Mindestens dre1 Arbettnehmer müssen diese Klage führen. Wir machen das stellvertretend für viele Kollegen hier um Werk."

Die Reaktion der Geschäftsleitung und des neugewählten Betriebsrats der »Mannschaft der Vernunft « auf diese Wahlanfechtung ließ nicht lange auf sich warten: Mit Schreiben vom 25.4.1984 wurden alle drei Antragsteller außerordentlich zum 26. 4. 84 gekündigt. Der Betriebsrat hatte hierzu seine Zustimmung erteilt. Begründet wurde die fristlose Kündigung damit, daß durch das verteilte Flugblatt der Betriebsfrieden gestört worden und außerdem die neugewählten Betriebsratsmitglieder ebenso in ihrer Ehre verletzt worden seien wie der Werksleiter, dem gezielte Wahlbeeinflussung unterstellt werde. Zudem wurde den Entlassenen untersagt, das Werksgelände von BMW zu betreten (Hausverbot).

\section{Die fehlende Unterstützung durch die Gewerkschaften}

Knirsch, Vollmer und Köbrich erhoben daraufhin beim Arbeitsgericht Berlin Kündigungsschutzklage und stellten den Antrag auf Weiterbeschäftigung zu den bisherigen Bedingungen. Auch außergerichtlich versuchten sie sich gegen ihre Entlassung zu verteidigen - ein Unterfangen, weiches sich insbesondere deshalb als schwierig erwies, weil die Ortsverwaltung der IG Metall demonstrativ auf Distanz zu ihnen ging. So verweigerte sie bezüglich der Wahlanfechtung Beitritt und Rechtsschutz und ließ sich hiervon auch nicht von einer Berliner IGM-Funktionärsversammlung vom 4. Mai 1984 abbringen. (Diese Entscheidung wurde erst im Januar 1985 vom Vorstand der IG Metall in Frankfurt revidiert, nachdem das AG Berlin bereits am 8.8.1984 der Wahlanfechtung in $\mathrm{r}$. Instanz stattgegeben hatte). Rechtsschutz für ihre Kündigungsschutzklage wurde den drei Entlassenen erst nach drei Wochen gewährt, ihr Antrag auf Gemaßregelten-Unterstützung für die achtwöchige Sperrfrist zwischen ihrer Kündigung und der erstmaligen Zahlung von Arbeitslosengeld wurde abgelehnt. Denn die Wahlanfechtung sowie deren Begründung in dem von ihnen erstellten und verteilten Flugblatt seien nicht im Auftrag der Organisation geschehen, der Kündigungsgrund beruhe folglich nicht auf einer gewerkschaftlichen Betätigung. In der Tat befand sıch die Ortsverwaltung in einem 
tiefergehenden Interessenkonflikt: Einerseits waren die Gekündigten für ihre jahrelange aktive Vertrauensleute- und Betriebsratsarbeit und ihr Engagement bei der Vorbereitung der um die Verkürzung der Wochenarbeitszeit auf 35 Stunden geführten Tarifauseinandersetzungen im Frühsommer 1984 bekannt. Daß sich der gewerkschaftliche Organisationsgrad bei BMW von $45 \%$ im Jahre 1979 auf über $65 \%$ bis Ende 1983 erhöhte, wird wohl nicht zu Unrecht auf die konfliktorische Betriebsrats- und Gewerkschaftspolitik zurückgeführt, deren hauptsächliche Repräsentanten sie waren. Andererseits wurden dadurch zwangsläufig die Spannungen innerhalb des Betriebsrats verschärft. Die von diesem Gremium bis Ende der siebziger Jahre einhellig verfolgte Politik einer kooperativen Form der Interessenvertretung gegenüber der Geschäftsleitung sah sich personell und sachlich mit einer Alternative konfrontiert, die zwar nur von einer - freilich relativ starken - Minderheit in der Belegschaft unterstützt wurde, diesen Nachteil jedoch durch ihre starke Verankerung im gewerkschaftlichen Vertrauensleutekörper und ihr großes Engagement wettmachte. So kam es zu einer deutlichen Fraktionierung im Betriebsrat, dessen Majorität sich zunehmend in die Defensive gedrängt sah.

Die Ortsverwaltung suchte in diesem Konflikt zu vermitteln: Daher ihr schließlich allseits akzeptierter Vorschlag, bei den Betriebsratswahlen vom April 1984 eine reine Persönlichkeitswahl durchzuführen und nicht etwa eine Listenwahl, bei der die drei Gekündigten mit Sicherheit ein Betriebsratsmandat erhalten hätten. Sie standen nämlich auf der Absicherungsliste des Vertrauenskörpers der IG Metall, welche für den Fall aufgestellt worden war, daß unmittelbar vor Ablauf der Frist zur Listeneinreichung eine oder mehrere weitere Listen eingereicht würden, ganz obenan (Plätze 1, 2 und 5). Nun hatte, womit niemand rechnen konnte, die selbsternannte Fraktion der "Mannschaft der Vernunft" alle is Sitze erreicht zweifellos ein eindeutiges Votum der Belegschaftsmehrheit. Und da von den is Gewählten allein I3 ebenfalls der IG Metall angehörten, war das Dilemma für die Ortsverwaltung perfekt: Entweder sie unterstützte den Betriebsrat mit dem Risiko, bei erfolgreicher Wahlanfechtung der Kollaboration mit unternehmerfreundlichen, wenn nicht -abhängigen Belegschaftsvertretern bezichtigt zu werden, oder sie setzte auf die Karte der Unterlegenen mit dem Risiko, ihren institutionellen Einfluß im Betrieb zu verlieren. Die IG Metall Berlin entschied sich zugunsten der faktischen Machtverhältnisse, für die "Mannschaft der Vernunft «.

Die drei Gekündigten standen folglich zunächst weitgehend isoliert da. Gleichwohl gelang es, vor allem dank des alsbaid ins Leben gerufenen »Solidaritätskomitees für die entlassenen BMW-Gewerkschafter ", den Vorgang publik zu machen und die Aufmerksamkeit der Öffentlichkeit auf ihn zu lenken. Eine Anzahl von Betriebsund Personalräten aus Berlin gaben Solidaritätserklärungen zugunsten der Gekündigten ab, im Mai 1984 berichtete erstmals die Presse, dann auch der Sender Freies Berlin über den Fall, wobei auch einer der Entlassenen selbst zu Wort kam. Außerdem informierten die Entlassenen per Flugblatt die Belegschaft über ihre Kündigung sowie die Tatsache, daß infolge ihres besonderen gesetzlichen Kündigungsschutzes »nur die ausdrückliche Zustimmung des Betriebsrats zur fristlosen Entlassung führen « konnte: „Die Folge der Zustimmung des Betriebsrats ist unsere Entlassung «, heißt es darin wörtlich.

\section{Die Kettenkündigungen}

Diese Vorgänge nahm die Geschäftsleitung von BMW zum Anlaß, das Arbeitsverhältnis der beiden ehemaligen Betriebsräte Knirsch und Vollmer mit Schreiben vom 30. 5. 1984 erneut außerordentlich zum 3.Junt 1984 wegen »wiederholter, in öffent- 
lichen Medien durchgeführter Ehrverletzung des Betrıebsrates und der Betriebsleitung, anhaltender Störung des Betriebsfriedens sowie anhaltender Schädigung des Ansehens der BMW-AG in der Öffentlichkeit« zu kündigen. Wiederum hatte der neugewählte Betriebsrat ohne Anhörung der Betroffenen dieser Maßnahme zugestimmt. Doch BMW beließ es nicht bei diesen beiden Kündigungen: Zum 20. September 1984 wurden gegen die drei Gewerkschafter erneut "rein vorsorglich" weitere fristlose Kündigungen ausgesprochen mit der Begründung, Knirsch und Vollmer hätten in Briefen an BMW-Beschäftigte eine Adressenliste der Firma (sog. Alpha-Liste) verwendet, die sie sich nur widerrechtlich hätten aneignen können. Das gleiche gelte auch für eine Protokollkopie über die Betriebsversammlung vom 27.3.1984. Außerdem hätten sie über das »angebliche "Solidaritätskomitee Flugblätter verteilen lassen, durch die der Betriebsfrieden nicht unerheblich beeinträchtigt worden sei. Und schließlich hätten sie den amtierenden Betriebsräten, die vor den Betriebstoren Flugblätter der IG Metall verteilten, diese entrissen und selbst »aggressiv verteilt«. Dabei habe zudem Köbrich Kollegen bei der Zufahrt zum Betriebsgelände behindert.

Mit Schreiben vom 9. 10. 1984 werden Knirsch und Vollmer abermals zum II. IO. 1984 vorsorglich fristlos gekündigt, weil sie im Zusammenhang mit der Übergabe der Betriebsratsämter unbefugt Kisten und Pakete aus dem Werk entfernt hätten.

Am 26. 10. 1984 erhielt Köbrich seine dritte, diesmal fristgerechte ordentliche Kündigung, auch diese wiederum rein vorsorglich für den Fall, daß sich die beiden ersten Kündigungen vor Gericht als unwirksam erweisen sollten. Da Köbrich nicht bereits früher Betriebsratsmitglied war, kam er lediglich in den Genuß des halbjährigen nachwirkenden Kündigungsschutzes nach $\$$ I 5 Abs. 3 KSchG, während seine Kollegen gemäß Abs. I dieser Vorschrift für ein Jahr Kündigungsschurz genossen.

Am 24.4.1985 wurde gegen Vollmer die fünte außerordentliche und zugleich erstmals eine ordentliche Kündigung ausgesprochen mit der Begründung, im Werk sei es wegen seiner in Aussicht stehenden Weiterbeschäftigung zu einer "spontanen Arbeitsniederlegung « gekommen.

Am 24. 5. 1985 wurden alle drei Betroffenen abermals gekündigt, weil sich Beschäftigte von BMW gegen ihre Weiterbeschäftigung ausgesprochen hätten.

Innerhalb von wenig mehr als einem Jahr sind damic Vollmer siebenmal, Knirsch fünfmal und Köbrich viermal gekündigt worden, wobei der amtierende Betriebsrat der "Mannschaft der Vernunft« den Kündigungen jeweils ohne Anhörung der Betroffenen teils ausdrücklich zugestimmt, 'zumindest aber nïkht widersprochen hat. Diese permanent wiederholten Kündigungen von Arbeitnehmern, die ja längst, seit der Kündigung, aus dem Betrieb ausgeschieden waren, signalisieren das massive, der Unterstützung durch den neugewählten Betriebsrat gewisse Interesse des Betriebes, das Arbeitsverhältnis mit den drei unterlegenen Betriebsratskandidaten in jedem Fall zu lösen. Allerdings fragt sich, warum das Unternehmen hierfür die Strategie der Kettenkündigungen einschlägt, von denen doch naheliegend ist, daß sie nur zu diesem Zweck ausgesprochen wurden: Welchen materiell-rechtlichen bzw. prozessualen Vorteil erhält ein Arbeitgeber durch diese Vorgehensweise und wie können sich die Betroffenen dagegen zur Wehr setzen? Ist es insbesondere dadurch möglich, einen etwaigen Anspruch der Gekündigten auf Weiterbeschäftigung zu unterlaufen mit der Folge, daß sie dauerhaft den Kontakt zur Belegschaft und das Betätigungsfeld ihres gewerkschaftlichen Engagement verlieren? Wie wirkt sich ferner die Tatsache aus, daß die Kündigungen in engem tatsächlichen Zusammenhang mit der Wahlanfechtung erfolgten, die auf den Vorwurf einseitiger unternehmerischer Einflußnahme zugunsten des neugewählten Betriebsrats gestützt wurde? 
Durch Beschluß des Arbeitsgerichts Berlin vom 8.8. 1984 (Az: I8 BV 5/84) wurde die Betriebsratswahl vom 2. und 3 . April 1984 bei BMW für unwirksam erklärt. Das Landesarbeitsgericht Berlin hat inzwischen durch Beschluß vom 6. 3. 1985 (Az: $6 \mathrm{Ta}$ BV 8/84) diese Entscheidung bestätigt und die Beschwerde der Firma sowie des Betriebsrats hiergegen zurückgewiesen, gleichzeitig jedoch die Rechtsbeschwerde zum BAG zugelassen: Deshalb ist der neugewählte Betriebsrat noch immer im Amt und juristisch in der Lage, an Arbeitgeberentscheidungen zuungunsten derjenigen mitzuwirken, die ihm a) die Rechtmäßigkeit dieser Amtsposition - mit offenbar nicht willkürlich aus der Luft gegriffenen Argumenten - bestreiten und zu deren loyaler Interessenvertretung er b) als Repräsentant der Belegschaft verpflichtet ist.

\section{r. Unzulässtgkeit wegen Kündigung der Antragsteller?}

Ein besonderes Problem der Zulässigkeıt des Anfechtungsantrags ergab sich daraus, daß diese nach der Anfechtung der Betriebsratswahl - wegen der betriebsöffentlichen Begründung derselben - gekündigt worden waren: Entfällt dadurch nachträglich ihre Antragsberechtigung, deren Fortbestand während der gesamten Verfahrensdauer nach der Rechtsprechung des BAG (zuletzt v. 10.6. $1983=\mathrm{AP} \mathrm{Nr}$. IO zu \I9 BetrVG) erforderlich ist, so daß mangels einer Übernahme des Anfechtungsantrags durch eine der im Betrieb vertretenen Gewerkschaften (IGM und DAG) der Antrag als unzulässig zurückgewiesen werden müßte? Oder war es zumindest erforderlich, das Anfechtungsverfahren bis zum rechtskräftigen Abschluß der Kündigungsstreitigkeiten auszusetzen? Diese bisher in der Rechtsprechung noch nicht entschiedenen Fragen wurden vom ArbG und LAG Berlin zugunsten der eigenständigen Durchführung und Entscheidung des Anfechtungsverfahrens trotz der erfolgten Kündigungen gegenüber den Anfechtungsberechtigten beantwortet, was zumindest implizit die Revision einer der folgenden drei Prämissen voraussetzt: Entweder die Kopplung der Antragsberechtigung an die Wahlberechtigung (gesetzliche Prämisse des $\$ 19 Abs. 2 BetrVG) oder die Verknüpfung des aktiven Wahlrechts mit dem rechtsgültigen Fortbestand des Arbeitsverhältnisses (mit Ausnahme der wegen Verstoßes gegen $₫ 102$ Abs. I BetrVG, $₫ 9$ MuschG oder $₫$ I 2 SchwbG offensichtlich rechtsunwirksamen Kündigung sowie einer nach $₫ 102$ Abs. 5 BetrVG oder aus sonstigen Gründen bestehenden Weiterbeschäftigungspflicht, Prämisse der b.M.), oder das Erfordernis des Fortbestands der Anfechtungsbefugnis für die gesamte Dauer des arbeitsgerichtlichen Beschlußverfahrens (BAG-Prämisse). Alle drei Voraussetzungen als gültig anzuerkennen und in vorliegendem Fall doch zu dem skizzierten Ergebnis - welches von der Sache her das einzig richtige sein kann - zu gelangen, gleicht dem Kunststück einer Quadratur des Kreises: Zwar standen Knirsch und Vollmer als ehemalige Betriebsratsmitglieder für ein Jahr, alle drei Betroffenen als Kandidaten zur Betriebsratswahl für sechs Monate unter dem besonderen gesetzlichen Kündigungsschutz nach $\$$ is Abs. I bzw. 3 KSchG, jedoch wurde dieser durch die fristlose Kündigung des Arbeitgebers unterlaufen mit der Konsequenz, daß eine Weiterbeschäftigungspflicht kraft Gesetzes $(\$ 102$ Abs. $\$$ BetrVG) oder wegen gesetzlich statuierter Nichtigkeit der Kündigung nicht bestand. Folglich hätten sie bis zur gerichtlichen Anerkennung eines Weiterbeschäftigungsanspruchs kein aktives Wahlrecht besessen, so daß es zumindest vorübergehend an der Antragsbefugnis gefehlt hätte.

Gleichwohl wäre ein solches Ergebnis »unmöglich «: Denn der Arbeitgeber hätte es in der Hand, dem Anfechtungsverfahren von Betriebsratswahlen, wie hier gesche- 
hen, durch gezielte Entlassungen die Grundlage zu entziehen; wobei er bei dem hier vorgebrachten Anfechtungsgrund typischerweise auf ein gleichgerichtetes Interesse des gewählten Betriebsrats an der Abwendung des Anfechtungsverfahrens rechnen kann, so daß im Ergebnis die faktische wie normative Geltung des $₫$ I9 Abs. 2 S. 1 BetrVG vom Willen derjenigen abhinge, deren Respekt vor den Grundsätzen über das betriebsverfassungsrechtliche Wahlverfahren ja gerade angezweifelt wird.

Da im übrigen auch der Ausweg einer Aussetzung des Wahlanfechtungsverfahrens bis zum rechtskräftigen Abschluß der Kündigungsschutzstreitigkeiten nicht in Betracht kommen kann, da dies - selbst wenn man die hier eingeschlagene Praxis der Kettenkündigungen außer acht läßt -

"faktisch der Versagung eines effektiven Rechtsschutzes gleich(käme)«, weil „bei Ausschöpfung des Instanzenzuges in den Kündigungsstreitigkeiten rechtskräftıge Entscheidungen erst so spät vorliegen würden, daß eine danach ergehende Entscheıdung in dem vorliegenden Anfechtungsverfahren erst in Rechrskraft erwachsen könnte, nachdem die Amtszeıt des Betriebsrats bereits abgelaufen sein würde, so daß das Beschlußverfahren mit an Sicherhert grenzender Wahrscheinlichkeit schon durch Zettablauf gegenstandslos würde" (AG Berlin, S. 23 f.),

sahen sich das ArbG und LAG Berlin um einer in der Sache willen vertretbaren Entscheidung zur vorsichtigen Revision einer der oben genannten drei Prämissen gezwungen.

Das ArbG Berlin geht davon aus, $d a ß$ durch die Kündigungen die Antragsberechtigung nach $\$ 19$ Abs. 2 S. I BetrVG nicht beseitigt werde. Dies folge zum einen aus der »Ausgestaltung der Kündigungsschutzklage als Feststellungs- und nicht GegenGestaltungsklage. Der Arbeitnehmer begehrt nämlich nicht die Veränderung eines bis zum Urteil bestehenden Rechtszustandes, sondern die Aufrechterhaltung der Unwirksamkeit der Kündigung wegen deren Sozialwidrigkeit«(S. I I), zum anderen daraus, daß »nur eine schnelle Entscheidung effektiven Rechtsschutz verspricht « (ebd.). Sodann fährt es, unter deutlicher Anspielung auf die konkrete Fallkonstellation fort:

„Daran ändert auch die vom Bundesarbeitsgericht erwähnte, aber noch nucht entschiedene Möglichkeit des Eintritts eines Arbeıtnehmers in das schwebende Verfahren (vgl. AP Nr.7 und $10 \mathrm{zu} \$ 19$ BetrVG 1972) nichts, da dies jedenfalls nach Ablauf der Anfechtungsfrist nicht mehr möglich ist (vgl. BAG Nr. ro a. a. O.). Aber auch dann könnte der Arbertgeber durch den Ausspruch weiterer Kündigungen das Anfechtungsverfahren zum Stillstand bringen, wenn er ein Interesse daran besäße, mit dem nach der angefochtenen Wahl amtierenden Betriebsrat zusammenzuarbeiten. Diese Gefahr bestünde zwar nıcht, wenn eine in dem Betrieb vertretene Gewerkschaft dem Anfechtungsverfahren als Antragsteller beiträte, jedoch führt diese Erwägung zu keinem anderen Ergebnis; denn die im Betrieb vertretenen Gewerkschaften können einerseirs durchaus ein Interesse an der Aufrechterhaltung des vorläufigen Wahlergebnisses besitzen, so daß sie das Anfechtungsverfahren schon aus diesem Grunde nıcht betreiben. Zum anderen führte dies zum faktıschen Wegfall der ersten Varıante des $\$ 19$ Abs. 2 BetrVG (Anfechrung durch mındestens drei Wahlberechtigte), wenn effektıver Rechtsschutz nur für den Fall des Beitritts emner Gewerkschaft zum Anfechtungsverfahren auf der Seite der Antragsteller gewährleistet wäre. Angesıchts dessen ist jedenfalls so lange vom Fortbestand der Antragsbefugnis gekündigter Arbeitnehmer auszugehen, wie enne rechtskräftıge klageabwe1sende Entscheidung im Kündigungsschutzverfahren nicht vorliegt« (S.12 f.).

Im Ergebnis ebenso konstatiert das LAG,

»daß es hier auf den rechtskräftig festgestellten Fortbestand der Arbettsverhältnisse der Antragsteller für die Frage der weiteren Durchführung des Verfahrens nicht ankommt « (S. 24), weil solange, wie hier - über den Fortbestand der Arbeitsverhältnısse des bzw. der die Anfechtung einleitenden Arbeitnehmer noch gestritten wird, ... noch nucht von einem endgültigen Wegfall der Anfechtungsbefugnis ausgegangen werden (kann) (S. 22).

Im Unterschied zum Arbeitsgericht folgert das LAG aus der spezifischen Fallkonstellation, daß aufgrund des rechtlich objektivierten Interesses an einer Überprüfung 
»des Wahlverfahrens schlechthin ( $\mathrm{S} .24$ ), welches in der Verfahrensmaxime des Amtsermittlungsgrundsatzes nach $₫ 83$ Abs. I ArbGG zum Ausdruck komme, der Begriff der Wablberechttgung eines Arbeitnehmers nach $\$>$ BetrVG als Voraussetzung für seine Teilnahme an der Wahl enger auszulegen sei als im Hinblick auf seine Wablanfechtungsbefugnis nach $\int 19$ Abs. 2 BetrVG:

»... denn der Kreıs der Wahlberechtigten muß für einen bestimmten Stichtag feststehen und kann unmöglich von einer u. U. erst viel später zu treffenden gerichtlichen Entscheidung in anhängigen Kündigungsschutzrechtsstreitigkeiten abhängig gemacht werden. Dieses Gebor der Rechtssicherheit und Eindeutigkeit ist aber nicht tangiert, wenn die Wahlberechtigung eines Arbeitnehmers nıchr für einen bestımmten Stichtag eindeutig feststehen muß, sondern die Wahlberechtigung - wie hier - für die Dauer eines u. U. sehr langen Zeitraumes in Frage steht. Standen die das Anfechtungsverfahren einleitenden Arbeitnehmer zum Zeitpunkt der Antragstellung in einem Arbeitsverhältnis und war daher die Antragsbefugnis einmal gegeben, dann ist solange von dem Fortbestand dieser Befugnis auszugehen, bis infolge rechtskräftiger Klageabweisungen in zwischenzeitlich anhängig gemachten Kündigungsschutzrechtsstreitigkeiten die Beendigung des (der) Arbeitsverhältnis(se) endgültig feststeht, bzw. bis die Arbeitsverhältnisse durch rechtskräftige Auflösungsurteile ( $\$ \$ 9$, Io $\mathrm{KSchG}$ ) ihr Ende gefunden haben. Durch eine Durchführung des Anfechtungsverfahrens in dem (unsicheren) Schwebezustand werden schutzwerte Interessen der übrigen Beteiligten nicht berührt bzw. nicht verletzt. Bestätigt sich nicht der erhobene Vorwurf, der vermeintlich die Wahlanfechtung begründen soll, bleibt es beı dem bisherigen Zustand, gleichgültig, wann über die Kündigungen der Arbeitsverhältnisse der Antragsteller rechtskräftige Entscheidungen gleich welcher Ergebnisse vorliegen werden. Sollten die Klagen der Antragsteller rechtskräftig abgewiesen werden, bevor eine die Unwirksamkeit der Wahl aussprechende - endgültige - Gerichtsentscheidung ergeht, ist dies gleichfalls ohne Bedeutung im Hinblick auf die eintretende Gegenstandslosigkeit des Beschlußverfahrens. Aber auch falls eine der Kündigungsschutzklagen rechtskräftig abgewiesen würde, nachdem bereits rechtskräftig über die Wahlanfechtung antragsgemäß entschieden worden Ist, werden schutzwerte Interessen der Beteiligten nicht berührt. Zum einen hat kein Beteiligter Anspruch darauf, daß ein gerichtlich festgestellter Verstoß gegen wesentliche Wahlvorschriften, der die Unwirksamkeit der durchgeführten Wahl herbeigeführt hat, sanktıonslos bleibt, wenn ein zulässiges Wahlanfechtungsverfahren erst einmal eingeleitet worden ist. Zum anderen ist zu bedenken, daß es den objektivierten Interessen der Belegschaft entspricht, nur einen ordnungsgemäß gewählten Betriebsrat zum Organ der Betriebsverfassung zu bestellen; es ist daher nicht ensichtig, von der Durchführung des vorliegenden Verfahrens Abstand zu nehmen, bis endgültig über den Fortbestand der Arbeitsverhältnısse der Antragsteller befunden worden ist " (S. $25 \mathrm{ff}$.).

Angesichts der in diesem Fall zum erstenmal so kraß deutlich gewordenen Verfahrensherrschaft derjenigen, deren u. U. rechtswidriges Zusammenspiel im Interesse des Funktionierens betrieblicher Mitbestimmung (und nicht etwa der individuellen Antragsteller) zur Überprüfung anstehen, lösen beide Gerichte die aufgezeigte Prämissenkollision dahingehend, daß sie den Fortbestand der Anfechtungsbefugnis trotz der schwebenden Kündigungsschutzstreitigkeiten bejahen. Allerdings sind die rechtskonstruktiven Wege verschieden. Das Arbeitsgericht geht auf den gesetzlich vorgegebenen Zusammenhang von Wahlberechtigung und Anfechtungsbefugnis nicht ein. Es kann daher nur dann zu dem gefundenen Ergebnis gelangen, wenn es entgegen der ganz allgemeinen Auffassung vom Fortbestand des Arbeitsverbältnisses während des schwebenden Kündigungsschutzprozesses (unabhängig von dessen Ergebnis, also der Wirksamkeit der Kündigung) ausgeht. Diese Abweichung von der herrschenden Arbeitsrechtsdogmatik, die die gesetzliche Ausgestaltung des Kündigungsschutzverfahrens auf ihrer Seite hat, erscheint dem LAG offenbar als zu riskant. Daher hält es sich hinsichtlich der Antragsbefugnis zum Zeitpunkt der Antragstellung am Erfordernis der aktiven Wablberechtigung nach $₫>$ BetrVG fest, modifiziert aber die BAG-Prämisse von deren notwendigen Fortbestand während der gesamten Dauer des Beschlußverfahrens: Es genügt, wenn die einmal gegebene Antragsbefugnis nicht dauerhaft und endgültig entfallen ist. Nach erfolgter, aber im Wege der Kündigungsschurzklage angegriffener Kündigung durch den Arbeitgeber 
steht sie gewissermaßen unter der auflösenden Bedingung der rechtskräftigen

Abweisung der Kündigungsschutzklage.

Dieser Entscheidung des LAG ist zuzustimmen. Denn in der Tat ist der Begriff der Wahlberechtigung keineswegs immer in gleicher Weise auszulegen, sondern verschieden je nach den im konkreten Kontext zu berücksichtigenden Interessen. So ist erwa auch im Hinblick auf das passive Wahlrecht, welches ebenfalls an die Voraussetzung der Wahlberechtigung geknüpft ist ( $\$ 8 \mathrm{Abs.} \mathrm{I} \mathrm{BetrVG:} \mathrm{"Wählbar} \mathrm{sind} \mathrm{alle}$ Wahlberechtigten ...«), allgemein anerkannt, daß dieses nicht durch eine Kündigung entfällt, über deren Wirksamkeit gerichtlich noch gestritten wird (DietzRichardi, BetrVG, $\$ 8$ RdNr. 10). Ebensowenig wie dort tritt beim Wahlanfechtungsverfahren, wie das LAG zutreffend ausgeführt hat, eine Verletzung schutzwerter Interessen der Beteiligten durch die Unsicherheit über den Ausgang des Kündigungsschutzprozesses ein. Konsequent wäre daher zu fragen, ob nicht das Erfordernis des Fortbestandes der Wahlberechtigung während der Dauer des Wahlanfechtungsverfahrens überhaupt fallen gelassen werden müßte, wie dies das Bundesverwaltungsgericht mit Beschluß vom 27.4.1983 (BVerwGE 67, I45ff.) für den Parallelfall der Anfechtung von Personalratswahlen gemäß $\$ 25$ des Personalvertretungsgesetzes für das Land Baden-Württemberg entschieden hat. Danach ist in Übereinstimmung mit der auch zum Wahlrecht zu den politischen Körperschaften vertretenen Auffassung hinsichtlich der Anfechtungsberechtigung allein darauf abzustellen, ob die Antragsteller zum Zeitpunkt der anzufechtenden Wahl wahlberechtigt waren. Solange sie das Anfechtungsverfahren weiter betreiben, entfällt ihr Rechtsschutzinteresse auch nicht dadurch, daß sie zwischenzeitlich aus der Dienststelle ausgeschieden und damit für künftige Wahlen nicht mehr wahlberechtigt sind, weil es nicht dem Einzelinteresse, welches eine persönliche Beschwer voraussetzt, sondern dem Allgemeininteresse an der Ordnungsmäßigkeit der Wahl des Personalrats dient.

Angesichts der früher insoweit abweichenden, in seiner jüngsten Entscheidung zum Wegfall der Anfechtungsberechtigung vom 12.2. 1985 (DB 85, 1799f.) allerdings ausdrücklich offengelassenen Auffassung des BAG sah sich das LAG nach $\$ 92$ Abs. I ArbGG veranlaßt, die Rechtsbeschwerde zum BAG zuzulassen.

\section{Die Beeinflussung der Betriebsratswabl durch BMW: Die Begründetheit der Wablanfechtung}

Wesentlich unproblematischer stellt sich für beide arbeitsgerichtlichen Instanzen die Frage der Begründetheit des Anfechtungsantrages dar: Es steht für sie außer Zweifel, daß der Arbeitgeber die Wahlentscheidung der Belegschaft zugunsten der "Mannschaft der Vernunft « beeinflußt und dadurch seine betriebsverfassungsrechtliche Neutralitätspflicht verletzt hat. Unstreitig hatte der BMW-Werksleiter Glas auf der Betriebsversammlung vom 26. und 27. März 1984, die unter anderem der Vorstellung der Kandidaten für die Betriebsratswahl diente, zum Bericht des Betriebsrats (Vortragende waren u.a. Knirsch und Vollmer) wie folgt Stellung genommen:

"Ich möchte zunächst ein paar Worte zu den Ausführungen des BR abgeben, ich habe selten so viele Halbwahrhesten gehört, wie bei Herrn Knirsch und Herrn Vollmer. Die beiden sind hier fehl am Platz, die sollten Politiker werden. Ich frage mich nur, ob sie dann im Landtag oder Bundestag die richtige Partei finden."

Sodann erklärte er u. a. weiter:

»Sie, liebe Mitarbeiterinnen und Mitarbeiter, sind mündige Bürger, Sie haben sich in den letzten 3 Jahren selbst eine Meinung bilden können. Ich bitte Sie, von Ihrem Wahlrecht 
Gebrauch zu machen. Sie können is Kandidaten wählen. Wenn Sie nicht zur Wahl gehen, kann eine Gruppe Mehrherten bekommen, die nucht Ihre Interessen vertrıtt. Bitte, bedenken Sie, Sie wählen nicht nur den Betriebsrat, sondern auch Ihren Verhandlungspartner mit der Gl. Und diese Verhandlungspartner können nur dann etwas für Sie erreichen, wenn sie realistısche Ziele und nıcht in erster Linie vordergründige Einzelinteressen verfolgen. Bei kompromißfähigen Verhandlungspartnern ist eın Erfolg für beide Seiten erreichbar und damıt können wır werterkommen. Unflexible Ideologen werden in solchen Verhandlungen eine Abfuhr erhalten.

Aus Sicht der Unternehmungslettung und des GBR kommt dieser BR-Wahl in Berlin eine zukunftsweisende Bedeutung zu. Alle bisherigen freıwilligen Leistungen, wie z. B. Sonderzahlung, Erfolgsbeteiligung sind nur der persönlichen Energie und dem persönlichen Einsatz der Gl zu verdanken. Ich habe mich hier persönlich eingesetzt, weil der GBR-Vorsitzende gar nicht so dafür war, daß̉ dies für Berlin bezahlt worden ist.

Natürlich will ich einen demokratisch gewählten BR, ich persönlich will keine Ja-Sager ım BR, ich bin der Meinung, daß wir emen BR brauchen, mit dem wir sachlich und fachlich verhandeln können.

Nachdem Sie bei dieser Wahl die Möglichkeit haben, durch die direkte Wahl der Kandidaten die Einstellung der Belegschaft zu BMW aufzuzeigen, liegt die Sicherheit der Arbertsplätze und die Zukunftssicherung der sozialen Leistungen direkt in Ihren Händen. Eine Stimmabgabe mit Überlegung und Verstand kann niemals zum Schaden von Belegschaft und Unternehmen sein. Der Ausgang der Wahl wird auch in München Entscheidungen für das Werk Berlin beeinflussen."

Nach dem Beitrag des Werkleiters erklärte der Vorsitzende des Wahlausschusses, daß er die Anwesenden bitte, die ersten drei Sätze der Ansprache des Werkleiters aus dem Gedächtnis zu streichen, er habe Angst, daß dadurch die Wahl angefochten werden könne. Hierauf erwiderte der Werksleiter:

„Ich nehme das nıcht zurück. Wenn Sie das als Wahlbeennflussung sehen, dann tut es mir leid. Ich habe zu dem Bericht des Herrn Knirsch und Herrn Vollmer gesagt, daß sie besser in der Politik aufgehoben wären als hier.«

Der vorbereitete Text des Redebeitrags des Werksleiters - mit Ausnahme des Einleitungssatzes - wurde der Belegschaft am nächsten Tag durch Auslegung im Betrieb zur Kenntnis gebracht.

Außerdem hatte das Unternehmen bei der Herstellung einer Wahlzeitung für die bei der Wahl erfolgreichen Kandidaten der »Mannschaft der Vernunft « technische Hilfe geleistet, indem die Fotos der Kandidaten von einer Public Relations-Abteilung der Unternehmenszentrale angefertigt wurden; und darüberhinaus hatte es auch die Druckkosten dieser Broschüre getragen. Diese Wahlzeitung, die zusätzlich zu den Abbildungen der Kandidaten auch einen ihre Vorstellungen erläuternden Text in deutsch, türkisch und serbokroatisch enthielt, wurde an den Wahltagen vor den Wahllokalen und vor dem Betrieb verteilt.

In beiden Vorfällen sahen ArbG und LAG Berlin eine unzulässige Beeinflussung der Betriebsratswahl, so daß diese wegen Verstoßes gegen die Bestimmung des $\ 20$ Abs. 2 BetrVG, nach der "niemand die Wahl des Betriebsrats durch Zufügung oder Androhung von Nachteilen oder durch Gewährung oder Versprechung von Vorteilen beeinflussen (darf) «, unwirksam ist. Diese Bestimmung konkretisiert das LAG in bezug auf den Arbeitgeber dahingehend,

"daß er sich strikt jeder Meınungsäußerung zu enthalten hat, die in Gestalt einer Wahlpropaganda Einfluß nehmen könnte für die Willensentscherdung zugunsten bestimmter Kandidaten. Die Betriebsratswahl dient der Legitimation der betneblichen Arbeitnehmerrepräsentanten, die im Verhältnis zum Arbeitgeber die Beteiligungsrechte der Belegschaft ausüben. Bei der Bildung der Arbeitnehmervertretung, der zukünftigen Partnerin im Rahmen der Betriebsverfassung, obliegt somit dem Arbettgeber ein von sich heraus selbstverständliches Neutralitätsgebor (Dietz-Richardi, BetrVG, $\mathbb{2 0}$ Anm. 18). Wie das Arbeitsgencht zu Recht ausgeführt hat, sind nämlich Bildung und Zusammensetzung des Betriebsrates ausschließlich eine Angelegenheit der Arbeitnehmer, was zwangsläufig bedingt, daß der Arbeitgeber als sein Gegenspieler sich jeglichen Einfluß auf dessen Zusammensetzung zu enthalten hat" (S. 28). 
Nach Auffassung beider Gerichte sind diese Grenzen der Einflußnahme auf die Wahlentscheidung der Belegschaft mit der auszugsweise zitierten Rede des Werksleiters und der Finanzierung und Herstellung der Wahlzeitung überschritten. Im Einzelnen führt das LAG (S. $30 \mathrm{ff}$.) hierzu aus:

„Bereits mit seınem Einleitungssatz hat er die Beteiligten zu 1) und 2) persönlich angegriffen und diffamırt; mit seınem Hinweis, sie sgehörten nicht hierhers meınte er offensıchtlich nıcht nur ihre Eigenschaft als Arbeitnehmer im Betrieb sondern auch die Mitgliedschaft im Betriebsrat; daß damıt auch auf die zukünftıge Zusammensetzung des Betriebsrates angespielt wurde, zeigt seıne Äußerung, die Beteiligten zu I) und 2) würden selbst in einern Parlament wohl keıne passende Parte1 finden, und sem Hinwers im wetteren Verlauf der Rede, unflexible Ideologen auf seiten des Betriebsrats würden in Verhandlungen mit der Geschäftsleitung eıne Abfuhr erhalten.

Mögen diese allgemeinen Ausführungen des Werksleiters vielleicht nur auf unachtsame Ausdrucksweise zurückzuführen seın, so sst doch auffällig, daß danach der Werksleıter in seıner Rede eine unmittelbare Verbindung der Beibehaltung der bisher gewährten Sozıalleistungen zu dem Ergebnıs der bevorstehenden Wahl herstellte, denn die von ihm angesprochenen Arbeitnehmer sollten durch >die direkte Wahl der Kandidaten` ihre ,Einstellung zu BMW aufzergen und durch ihre Stımmabgabe läge die Sicherheıt der Arbeitsplätze und die Zukunftssıcherung der sozialen Leistungen direkt $\mathrm{in}$ ihren Händen. Versteckt - aber für den aufmerksamen Zuhörer unüberhörbar - lag darin die Andeutung, u. U. könnte die Weitergewährung der Sonderzulage und Erfolgsbeteiligung in Gefahr geraten, falls die am Anfang der Rede persönlich angegriffenen Kandidaten in den neuen Betriebsrat gewähit würden. Diese Deutung der Worte des Werksleiters ist auch nıcht überzogen; zum Schluß führte er aus, daß ene Stimmabgabe mit Überlegung und Verstand niemals zum Schaden der Arbeitnehmer und des Unternehmens» sein werde. Da sıch auch eine Gruppierung von Kandidaten unter der Bezeıchnung "Mannschaft der Vernunft« zur Wahl stellte, mußte sıch für jeden Zuhörer der Eindruck aufdrängen, der Werksleiter meine mit einer Stimmabgabe mit Verstand eine Wahlentscheidung zugunsten dieser 'Mannschaft der Vernunft, und werde diese gewählt, seien die bisher erhaltenen Sozialleistungen eher gesichert.

Daß diese Verbindung zwischen einer Wiederwahl der Beteiligten zu I) und 2) und der Gefährdung der Sozıalleistungen, die in der Rede nur angedeuter wurde, tatsächlich aber auch so vom Werksleiter ernstlich gemeint war, zeıgen seıne Bekundungen, die er als Zeuge in dem Rechtsstreit Knursch ./. BMW -6 Ca 141784- am 2.10.1984 machte. Gemäß den yon der Beteiligten zu s) selbst überreichten Fotokopien der Sitzungsniederschrift (Bl. 317-320 d. A) hat er nämlich eıngeräumt, gegenüber dem Beteiligten zu I) am 30. März erklärt zu haben, er denke daran, die betrieblichen Sozıalleistungen zu kürzen, wenn der bisherige Betriebsrat wieder gewählt würde.

Hinzu kommt, daß der Werksleiter nach seiner Ansprache durch den Vorsitzenden des Wahlausschusses - Schultz - darauf hingewiesen worden 1st, daß seıne Einführungsworte im Hinblick auf die Wahl bedenklich seien, jedoch dann - erwidernd - ausdrücklich betonte, daß er nichts zurücknehme und seine Einleitungssätze sinngemäß wiederholte. Eine glerchwertıge Betonung der Beibehaltung seines Standpunktes findet sich auch in dem vor der Wahl für die Belegschaft ausgelegten Text seiner Rede vom 27. März, beı dem sich gleich zu Beginn der Hinweis findet: ,Es gilt in jedem Fall das gesprochene Wort; damit ist wieder auf die persönlichen Angriffe des Werksleiters gegen die Beteiligten zu 1) und 2) Bezug genommen." (S. $30 \mathrm{ff}$.

\section{Der Einfluß der Wablanfechtung auf die Stellung des Betriebsrats}

\section{a) Ketne Rückwirkung der Anfechtung}

Trotz der Evidenz und Intensität dieser Einflußnahme der BMW-Betriebsleitung auf die Betriebsratswahl 1984 zugunsten der extrem kooperationsgeneigten Kandidatengruppe, der hohen Wahrscheinlichkeit, daß dadurch die Zusammensetzung des neu gewählten Betriebsrats bedingt wurde, und der Tatsache, daß dies gleichlautend in zwei Beschlußinstanzen festgestellt worden ist, amtiert er nach wie vor. Seine Mirwirkung an den rechtsgeschäftlichen Handlungen des Arbeitgebers ist rechtsgültig und bleibt dies, auch wenn die Unwirksamkeit der Wahl rechtskräftig 
festgesteilt wird, weil die erfolgreiche Anfechtung nicht zur Folge hat, daß der fehlerhaft gewählte Betriebsrat rückwirkend sein Amt verliert.

Dieser zwar nicht gesetzlich fixierte Grundsatz wird mit dem Interesse am Bestand der Maßnahmen, an denen der Betriebsrat beteiligt ist, sowie mit dem Schutz der Betriebsratsmitglieder für die Dauer ihrer Amtstätigkeit legitimiert (vgl. DietzRichardi, BetrVb, $\$ 19$ RdNr. 53). Bei der im vorliegenden Fall gegebenen Interessenkonstellation, die typischerweise stets dann vorliegt, wenn eine Betriebsratswahl wegen unzulässiger Wahlbeeinflussung durch den Arbeitgeber angefochten wird, hat dies zur Folge, daß der Arbeitgeber, sofern er gegenüber denjenigen Arbeitnehmern, die die Wahl angefochten haben, die Kündigung ausspricht, auf ein gleichgerichtetes Interesse des Betriebsrats rechnen kann. Dieser wird demnach in aller Regel einer ordentlichen Kündigung nicht gem. $\$ 102 \mathrm{Abs.} 3$ BetrVG widersprechen, gegen eine außerordentliche keine Bedenken äußern ( $\$ 102$ Abs. 2). Daß er gar, wie im vorliegenden Fall zunächst geschehen, den Kündigungen ausdrücklich seine Zustimmung erteilt, beruhte auf der irrtümlichen Annahme aller Beteiligten, die Beseitigung des nachwirkenden Kündigungsschutzes nach $₫$ is Abs. I S. 2 und Abs. 3 S. 2 KSchG für ehemalige Betriebsratsmitglieder und Wahlbewerber erfordere wie nach $\ \mathrm{rO}_{3}$ Abs. I BetrVG die Zustimmung des Betriebsrats.

\section{b) Der Ausbau der innerbetrieblichen Machtpositzon des Betriebsrats trotz Wablanfechtung}

Bis zur rechtskräftigen Feststellung der Unwirksamkeit seiner Wahl bleiben die betriebsverfassungsrechtlichen Handlungskompetenzen des Betriebsrats folglich ungeschmälert erhalten. Dies erlaubt es ihm, wie der seitherige Verlauf der Auseinandersetzungen im BMW-Werk Berlin zeigt, ungeachtet der erwiesenen aktiven Wahlhilfe durch den Arbeitgeber, seine innerbetriebliche Machtposition zu erhalten und sogar auszubauen - zumindest solange, wie die betreffende Gewerkschaft nicht bereit oder in der Lage ist, in einem derartigen Konflikt eindeutig und entschieden Partei gegen ihn zu ergreifen. Die Ortsverwaltung der IG Metall hat zwar die "Mannschaft der Vernunft « nach dem Erfolg der Wahlanfechtung in I. Instanz erstmals am 29. 8. I984 öffentlich zum Rücktritt aufgefordert und dies inzwischen mehrfach mit Nachdruck bekräftigt. In krassem Widerspruch hierzu war sie ihr aber dabei behilflich, ihren Einfluß auf den gewerkschaftlichen Vertrauensleutekörper im Betrieb zu einer inzwischen eindeutigen Majorität auszubauen: Die gesamte Vertrauenskörperleitung besteht inzwischen aus Mitgliedern der "Vernunfffraktion «. In gleicher Weise unnachvollziehbar ist die unterschiedliche Handhabung der gestellten Ausschlußanträge: Der von den Mitgliedern der »Mannschaft der Vernunft" gestellte Ausschlußantrag gegen Knirsch und Vollmer aus der IG Metall führte zur Einleitung eines Untersuchungsverfahrens gegen Vollmer, welches über Monate betrieben und erst im Dezember 1984 eingestellt wurde. Dagegen hat die Ortsverwaltung die Entscheidung über die Einleitung eines entsprechenden Verfahrens gegen die zwölf IG Metallgewerkschafter des Betriebsrats noch im Januar 1985 bis zur rechtskräftigen Entscheidung über die Wahlanfechtung mit der Begründung abgelehnt, daß »die Auslegung des Betriebsverfassungsgesetzes nicht gewerkschaftsschädigend an sich « sei.

Inzwischen zeichnet sich aber eine deutliche Kehrtwende in der Haltung der IG Metall ab: Wie der Pressemitteilung des "Solidaritätskomitees für die entlassenen BMW-Gewerkschafter « vom 9. 10. 1985 zu entnehmen ist, hat die Gewerkschaft 
inzwischen beschlossen, den drei Gekündigten Gemaßregeltenunterstützung nach $\$ 24$ ihrer Satzung zu zahlen und diese Zahlung über die laut Satzung dafür vorgesehenen ersten I 3 Wochen hinaus zu verlängern. "Diese Entscheidung bedeutet«, so heißt es erläuternd in der Pressemitteilung, „daß die ursprünglich eingenommene Haltung, nach der die Drei bei ihrer Wahlanfechtung nicht im Auftrag< der Gewerkschaft gehandelt hätten, vollständig revidiert wird: Es ist jetzt nachträglich anerkannt, daß die - von der Berliner IGM-Ortsverwaltung nicht unterstützte Wahlanfechtungsinitiative der Drei eine gewerkschaftliche Aktivität war«. Diese Pressemitteilung berichtet ferner, daß die Berliner Ortsverwaltung der IG Metall beschlossen habe, ein Untersuchungsverfahren nach $\$ I I ihrer Satzung (Ausschlußverfahren) gegen den Vertrauenskörperleiter und freigestelltes Betriebsratsmitglied der "Mannschaft der Vernunft « einzuleiten, nachdem dieser einen Kollegen, der eine Beschwerde vorgebracht hatte, als Unruhestifter an die Personalabteilung weitergemeldet hatte.

Diese Korrektur der Position der IG Metall war überfällig. Freilich bleibt die Tatsache skandalös und irritierend, daß sie sich zu dieser moralischen und finanziellen Unterstützung ihrer gemaßregelten Mitglieder und Funktionäre erst bereit fand, nachdem sämtliche mit der Auseinandersetzung bislang befaßten Gerichte - auch in den Kündigungsschutzverfahren, über die in Teil II dieser Dokumentation berichtet werden soll - die Rechtswidrigkeit des Zusammenspiels von BMW und amtierendem Betriebsrat festgestellt hatten. Es wäre fatal, wenn die Gewerkschaften angesichts der wirtschafts- und arbeitsmarktpolitischen Defensive, in der sie sich ohnedies gegenüber den Unternehmern befinden, diese noch dadurch verstärkten, daß sie die Verteidigung elementarer Grundsätze des Betriebsverfassungsgesetzes allein den betroffenen Minderheiten und den Arbeitsgerichten überlassen: Eine solche Politik des praktischen Opportunismus gießt Wasser auf die Mühlen derjenigen, die aus ganz anderen Motiven als den propagierten Schlagworten von "Minderheitenschutz « und "Demokratisierung « auf die Schwächung der betrieblichen Interessenvertretung durch gesetzliche Veränderungen des Wahlverfahrens hinarbeiten. ${ }^{3}$

3 Zum $*$ Entwurf ennes Gesetzes zur Verstärkung der Minderheıtenrechte in den Betneben und Verwaltungen* (BT-Drs. 10/3384) aus den Fraktionen von CDU/CSU und FDP vgl. die kritischen Stellungnahmen von U. Mückenberger, Deregulierendes Arbeitsrecht, KJ H. $3 / 8,5.255$ ff. (266ff.) und B. BaumannCzichon, Der Etikettenschwindel mit dem Minderheitenschutz, in: R. Wahsner u.a., ’Heuern und Feuern^. Arbettsrecht nach der ,Wender (Hrsg.: Arbelterkammer Bremen), 1985, S. $188 \mathrm{ff.}$ 